\title{
Dielectric elastomers for wave energy harvesting
}

\section{Rocco Vertechy and Marco Fontana}

Inflatable deformable transducers promise effective and affordable devices to obtain energy sustainably.

Ocean-wave power is one of the most persistent, spatiallyconcentrated and predictable forms of intermittent renewable energies. The worldwide estimated resource amounts to nearly 3TW of yearly average power, and wave energy could cover a significant portion of the intermittent renewable energy mix in the future.

Harvesting energy from waves is very challenging and the sector is still immature, with only a few pre-commercial systems in operation around the world. Existing wave energy converters (WECs) are complex and costly to construct, install, and maintain. They are also vulnerable to the marine environment (experiencing large impulsive loads and corrosion) and show limited energy conversion efficiency.

In this context, dielectric elastomer generators (DEGs) could provide the technological breakthrough that is required to make wave energy exploitable. DEGs are deformable capacitors made with incompressible elastic dielectric layers and compliant electrodes that can be used to convert mechanical energy into electricity by variable capacitance electrostatic generation. ${ }^{1}$ Potential advantages of DEGs over conventional technologies are: large energy densities, direct-drive and cyclic operation, good and rate-independent efficiencies, good shock and corrosion resistance, silent operation, and moderate-to-low cost.

We have investigated different concepts for DEG-based $\mathrm{WECs}^{2-5}$ and consider the polymeric oscillating water column (poly-OWC) the most promising. It performs well at capturing energy, is architecturally simple, and is applicable on- and off-shore, as well as along the shoreline (see Figure 1). The poly-OWC is an upgraded version of the turbogenerator-based OWC converter, which is currently the most studied and best developed type of WEC.

The poly-OWC features a semi-submerged hollow structure (hereafter called 'collector') that is open at the sea bottom to the incoming wave field and closed at the top by an inflatable
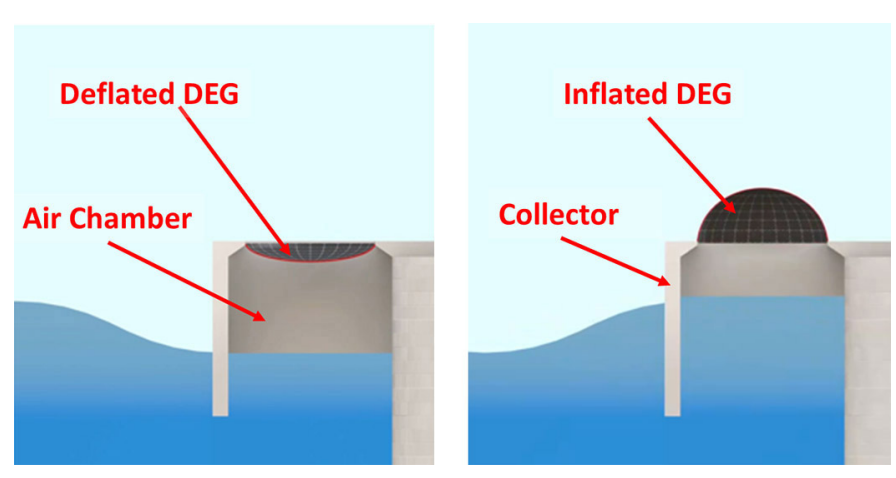

Figure 1. The working principle of a poly-oscillating water column (OWC) with fixed collector. DEG: Dielectric elastomer generator.

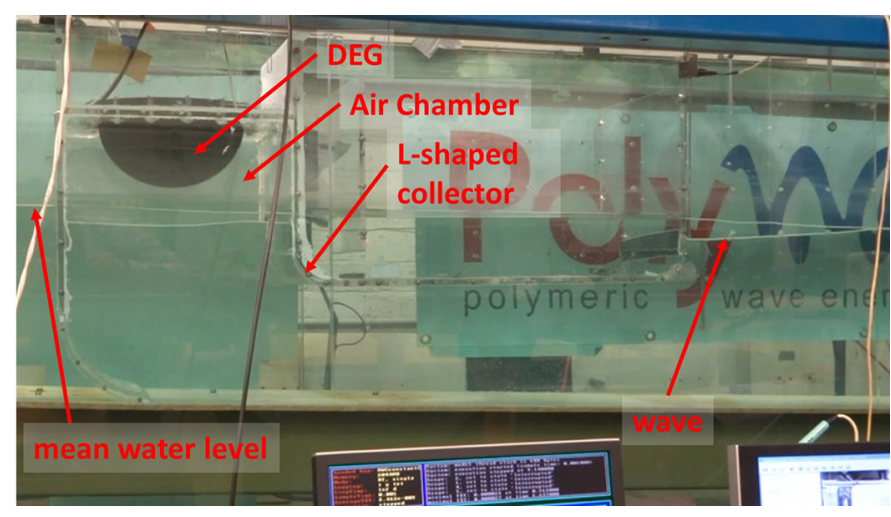

Figure 2. Two-dimensional wave flume testing of a poly-OWC with fixed L-shaped collector and rectangular cross section.

circular diaphragm DEG. The collector is partially filled with water (namely, the water column) and air. As waves break on the structure, the water column is put into motion, which compresses/expands the air and hence inflates/deflates the DEG. Regulation of the charge residing on the electrodes during DEG deformation makes it possible to control the forces acting on the water column and, thus, to extract energy from waves and convert it into electricity.

Continued on next page 
Numerical simulation studies ${ }^{4}$ have demonstrated that a fullscale poly-OWC system, with collector dimensions equivalent to those of the OWC Pico Plant (installed in the Azores), can potentially produce at least the same amount of energy as that obtainable from a turbogenerator-based system. However, the required inflatable DEG is expected to cost at most one fifth as much as the turbogenerator, so (at current material and manufacturing values) the energy would cost much less.

We experimentally validated the expected energy capturing abilities of the poly-OWC device with small-scale tests in wave tanks. ${ }^{5}$ We tested an optimized fully-functional 1:40 scale model in a 2D wave flume under monochromatic conditions (see Figure 2). The model consisted of a fixed OWC collector (L-shaped and with an upper rectangular cross-section with edges $260 \mathrm{~mm}$ and $360 \mathrm{~mm}$ long) fixed to the sea bed and an inflatable DEG $(125 \mathrm{~mm}$ in radius and $93 \mu \mathrm{m}$ in thickness) made with an acrylic elastomeric dielectric and conductive grease electrodes. The system was capable of harvesting energy with an average power of $670 \mathrm{~mW}$ for water waves with $4.5 \mathrm{~cm}$ height and $0.7 \mathrm{~Hz}$ frequency with a wave-to-wire

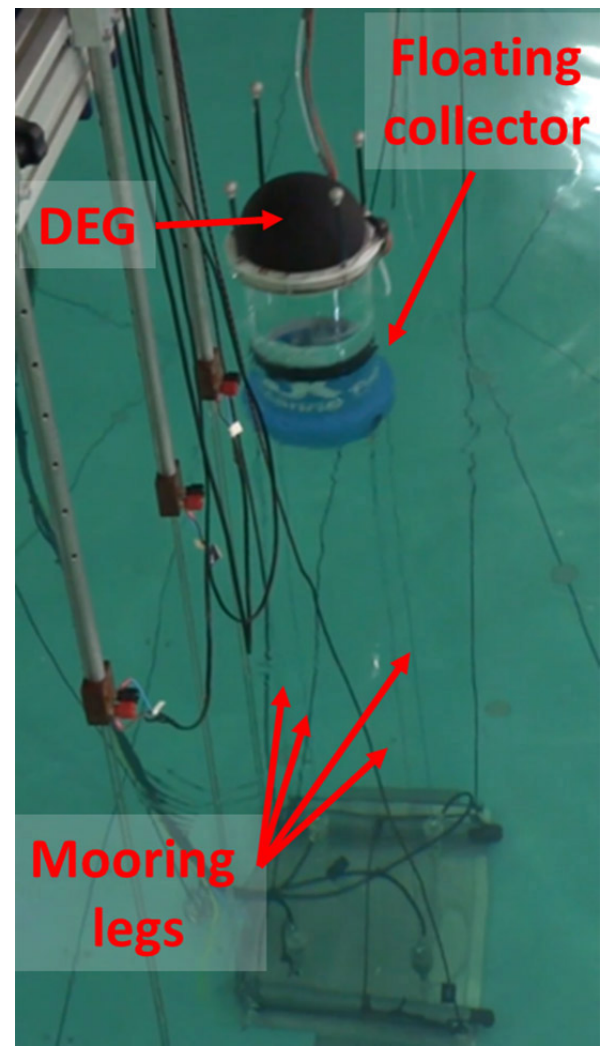

Figure 3. Three-dimensional wave-tank testing of a poly-OWC with floating tubular collector. efficiency close to $20 \%$. Energy density for the DEG was up to $109 \mathrm{~J} / \mathrm{kg}$. (That corresponds to an equivalent full-scale system average power of $270 \mathrm{~kW}$ for waves with $1.8 \mathrm{~m}$ height and $9 \mathrm{~s}$ period.) These results were confirmed by experiments on a floating 1:50 scale poly-OWC model in a 3D circular wave tank (see Figure 3) which also demonstrated the functionality of the system under irregular sea conditions.

We are now developing a larger 1:35 scale model of the floating poly-OWC system for long-term tests in a 3D wave tank to assess scalability issues. We also plan to investigate lifetime performance and degradation issues of DEGs operating in marine environmental conditions. Additionally, we shall identify processes and procedures for manufacturing large DEGs (tens of square meters in area and of centimeters in thickness). Finally, we are also expecting to develop, deploy, and test small-scale (1:5 to 1:10) poly-OWC devices in benign sea test sites.

This research was undertaken as part of the European Union Seventh Framework Programme project PolyWEC (www.polywec.org) under grant agreement 309139.

\section{Author Information}

\section{Rocco Vertechy}

Department of Industrial Engineering

University of Bologna

Bologna, Italy

Rocco Vertechy is an associate professor. His current research is mainly focused on mechatronic systems based on smart materials and compliant mechanisms.

\section{Marco Fontana \\ Sant'Anna School of Advanced Studies \\ Pisa, Italy}

Marco Fontana is an assistant professor and leads the 'PERCRO-SEES' group focusing on mechatronics and smart materials for renewable energy technologies.

\section{References}

1. R. Pelrine, R. Kornbluh, J. Eckerle, P. Jeuck, S. Oh, Q. Pei, and S. Stanford, Dielectric elastomers: generator mode fundamentals and applications, Proc. SPIE 4329, pp. 148 156, 2001. doi:10.1117/12.432640

2. G. Moretti, M. Fontana, and R. Vertechy, Modeling of a heaving buoy wave energy converter with stacked dielectric elastomer generator, Proc. Conf. Smart Mater. Adapt Struct. Intellig. Syst., pp. SMASIS2014-7565, 2014. doi:10.1115/SMASIS2014-7565 3. G. Moretti, D. Forehand, R. Vertechy, M. Fontana, and D. Ingram, Modeling of an oscillating wave surge converter with dielectric elastomer power take-off, 
Proc. 33rd Int'l Conf. Ocean, Offshore Arctic Eng., pp. OMAE2014-23559, 2014. doi:10.1115/OMAE2014-23559

4. R. Vertechy and M. Fontana, Reduced model and application of inflating circular diaphragm dielectric elastomer generators for wave energy harvesting, ASME J. Vibrat. Acoust. 137 (1), p. 011004, 2015. doi:10.1115/1.4028508

5. R. Vertechy, M. Fontana, G. P. Rosati Papini, and D. Forehand, In-tank tests of a dielectric elastomer generator for wave energy harvesting, Proc. SPIE 9056, p. 90561G, 2014. doi:10.1117/12.2045046 